No. 2, Volume 21, of the Jonirnal of General Microbiology was issued on 14 December 1959

Humphrey, B. A. \& Vincent, J. M. (1959). J. gen. Microbiol. 21, 477-484

\title{
Extracellular Polysaccharides of Rhizobium
}

\author{
BY BEVERLEY A. HUMPHREY AND J. M. VINCENT \\ Faculty of Agriculture, University of Sydney, Australia
}

\begin{abstract}
SUMMARY: The extracellular polysaccharides of seventeen strains (four species) of Rhizobium were studied chromatographically. The seven strains from lucerne (R. meliloti) produced polysaccharides which contained glucose but consistently lacked glucuronic acid. The remaining ten strains, which were isolated from pea (R. leguminosarum), clover (R. trifolii) and bean (R. phaseoli), produced a polysaccharide in which both glucose and glucuronic acid were demonstrated. These latter strains could be grouped into three types according to their glucose : glucuronic acid ratio, but such groups did not correspond with the species.
\end{abstract}

In 1931 Hopkins, Peterson \& Fred identified glucuronic acid in the extracellular polysaccharide of strains of Rhizobium meliloti, $R$. leguminosarum and $\boldsymbol{R}$. trifolii. Schluchterer \& Stacey (1945) found that the polysaccharide from a clover strain consisted of a glucose-glucuronic acid chain. Contrary to these findings, De Leizaola \& Dedonder (1955) found rhamnose, glucose, galactose, mannose, xylose and arabinose in preparations from four species of Rhizobium (pea, soya bean, clover, lupin), but reported complete absence of glucuronic acid. Kleczkowski \& Kleczkowski (1952) reported having found fructose in a clover strain, as well as glucose and glucuronic acid in this and in a strain of $\boldsymbol{R}$. leguminosarum. By using antisera developed against the parent bacterium, these workers obtained precipitation of antibody with the polysaccharide prepared from $\boldsymbol{R}$. leguminosarum. With $\boldsymbol{R}$. trifolii the polysaccharide did not cause precipitation in the homologous antiserum.

In the present investigation; polysaccharides from a selection of Rhizobium strains representing four species have been examined: $(a)$ chromatographically, with a view to investigating the report of De Leizaola \& Dedonder (1955); (b) quantitatively, by methods of spectrophotometric analysis, to determine their glucose: glucuronic acid ratio.

\section{METHODS}

Organisms and methods of culture. The following organisms were selected for study: Rhizobium trifolii (clover strains) SU 36, SU 46, SU 64, SU 91, SU 298/ 533, NA 30, NZ19; $R$. leguminosarum (pea strains) SU 302, SU 331; $R$. phaseoli (bean strains) NA 1; R. meliloti (lucerne strains) SU 27, SU 47, SU62, SU 74, SU 76, SU 101, SU 277. (SU refers to strains from the Sydney. University collection; NA, New South Wales Department of Agriculture; NZ, the Department of Scientific and Industrial Research, New Zealand.)

The organisms were inoculated into the medium formulated by Norris (1958) which contains (g./1.) mannitol, 10; disodium hydrogen phosphate, $0 \cdot 45$; magnesium sulphate heptahydrate; 0.1 ; potassium nitrate, 0.6 ; ferric chloride, 0.01; thiamin and biotin, trace. For each strain, four 1.l. flasks 
containing $100 \mathrm{ml}$. medium were inoculated and incubated at $26^{\circ}$ for 14 days. The gum was prepared by centrifuging down the organisms at $800 \mathrm{~g}$, and pouring the supernatant fluid, usually viscous and opalescent, into three volumes of ice-cold ethanol. With copious gum-producers strings of gum formed immediately; with other strains, the gelatinous precipitate of gum appeared only on standing overnight at $4^{\circ}$. The initial precipitate was centrifuged down, redissolved in water, again centrifuged to remove debris, and the supernatant fluid precipitated with three volumes of ethanol, usually slightly acidified with hydrochloric acid to coagulate the gelatinous precipitate. This twiceprecipitated material, washed with ether and dried in vacuo, was used for most analyses. 'Gum' in this paper refers to these relatively crude polysaccharide (total carbohydrate) preparations which still contained protein and ash.

Hydrolysis of gum for chromatography. Gum (10 mg.) and $0.5 \mathrm{ml}$. N-HCL were sealed in an ampoule and heated at $100^{\circ}$ for $20 \mathrm{hr}$. The hydrochloric acid was removed in vacuo over potassium hydroxide pellets and sulphuric acid, and the residue dissolved in an appropriate volume of water.

Chromatography. Descending paper chromatography was used with butanol + acetic acid + water $(4+1+5)$ and butanol + ethanol + water $(5+1+4)$ as solvents. $p$-Anisidine hydrochloride was used as developer for carbohydrates, and ninhydrin for amino acids. All identifications of spots were based on comparisons with authentic sugar samples.

Glucuronic acid analysis. This was done according to Bowness (1957) except that the $0.1 \%(\mathrm{w} / \mathrm{v})$ carbazole reagent recommended by Dische (1947) was used instead of the $\mathbf{0 . 2} \%$ carbazole reagent used by Bowness. The more dilute reagent gave a lower blank reading and more consistent results. Analysis for glucuronic acid was carried out on samples (containing $250 \mu \mathrm{g}$. gum) of the solution of unhydrolysed gum used also for glucose analysis. Replicates were precise to $\pm 5 \%$. Standard curves for glucuronic acid (10-50 $\mu \mathrm{g}$; Light \& Co.) and glucose (50-200 $\mu \mathrm{g}$.) were made with each batch of analyses. Absorption by glucose at $530 \mathrm{~m} \mu$ was less than $10 \%$ that of glucuronic acid. Before calculating the amount of glucuronic acid present, absorption by the gum solution was corrected for the amount of glucose found by anthrone analysis. Glucuronic acid added to a gum solution was $100 \%$ recoverable. Glucose and glucuronic acid gave additive absorption. Ashwell (1957) has stated that protein below $0.1 \%(\mathrm{w} / \mathrm{v})$ does not interfere with the carbazole reaction.

Glucose analysis. It was not found possible to obtain reproducible results for glucose analysis by the carbazole method of Bowness (1957). The anthrone method of Seifter, Dayton, Novic \& Muntwyler (1950) was used on solutions of unhydrolysed gum. Replicates precise to $\pm 1 \%$ were obtained on samples containing $100 \mu \mathrm{g}$. gum. A standard curve for glucose $(50-150 \mu \mathrm{g} . / 5 \mathrm{ml}$. solution) and for glucuronic acid (10-50 $\mu \mathrm{g} . / 5 \mathrm{ml}$. solution) was made with each batch of analyses. Glucuronic acid always gave an absorption of less than $5 \%$ that of glucose at $620 \mathrm{~m} \mu$; when necessary a correction for the glucuronic acid content of the gum was made.

Nitrogen analysis. Digestion by sulphuric acid and peroxide was followed by nesslerization. 
Deproteinization. A protein fraction could be removed from the twiceprecipitated gum by the deproteinization technique of Sevag, Lackman \& Smollens (1935). It was also found that drying the gum in vacuo rendered part of it insoluble in water. This insoluble fraction did not contain carbohydrate and was probably denatured protein.

Spectrophotometry. A Hilger Uvispek was used throughout.

\section{RESULTS}

Yield of gums and constituent sugars

The strains of Rhizobium used differed in the quantity of gum produced; the results with chromatography are also shown in Table 1 . In addition to the sugars identified in Table 1, all hydrolysates showed a small slow-moving spot, thought to be a di- or oligo-saccharide, possibly the cellobiuronic acid unit described by Schluchterer \& Stacey (1945). A fast-moving substance $\left(\boldsymbol{R}_{\text {glacose }}\right.$ $\mathbf{2 \cdot 0 - 2 \cdot 5}$ in butanol + acetic acid + water), which developed a bright pink coloration with $p$-anisidine hydrochloride in the cold, appeared with gum from all strains of Rhizobium trifolii except NA30. This substance was not xylose, as would be expected if it arose from decarboxylation of glucuronic

Table 1. Amount of twice-precipitated gum produced by different strains of Rhizobium, and constituent sugars detected by chromatography

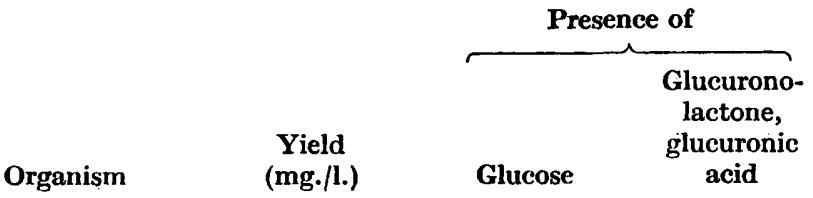

R. trifolii

SU36

SU 46

SU 64

SU91

SU 298/533

NA 30

(mg./1.)

NZ19

R. leguminosarum

SU 831

R. phaseoli

NA 1

R. meliloti 
acid during hydrolysis. Its spot does in fact correspond with that of 4-o-methyl glucuronic acid from beech hemicellulose.'

With ninhydrin developer, hydrolysates showed a similar pattern of eight. amino acids (or groups of amino acids). These were still present after one cycle of deproteinization by Sevag's method. Amino acids were almost all absent from the gum of Rhizobium trifolii SU 298/533 which had been separated from the water-insoluble fraction obtained after drying and then precipitated for a third time. Glucosamine was absent in all cases.

Solutions of the gums gave no coloration with iodine.

It was shown that replacement of mannitol in the culture medium by arabinose, glucose, xylose or sucrose as carbon source did not cause any, alteration. in the sugars present in the gum of Rhizobium trifolii $\mathrm{SU} 298 / 533$.

Table 2. Quantitative analysis of Rhizobium gums

\begin{tabular}{|c|c|c|c|c|}
\hline \multirow[b]{2}{*}{$\begin{array}{l}\text { Source of gum* and } \\
\text { treatment }\end{array}$} & \multicolumn{3}{|c|}{ Composition of gum } & \multirow[b]{2}{*}{$\begin{array}{c}\text { Glucuronic } \\
\text { acid in total } \\
\text { carbohydrate } \\
(\%)\end{array}$} \\
\hline & $\begin{array}{l}\text { Nitrogen } \\
(\%)\end{array}$ & $\begin{array}{l}\text { Total } \dagger \\
\text { carbohydrate } \\
(\%)\end{array}$ & $\begin{array}{l}\text { Glucuronic } \\
\text { acid } \ddagger \\
(\%)\end{array}$ & \\
\hline \multicolumn{5}{|l|}{$\boldsymbol{R}:$ :trifolii } \\
\hline SÜ36 & - & $41 \cdot 8$ & $9 \cdot 8$ & $23 \cdot 5$ \\
\hline SU 46 & - & $56 \cdot 3$ & $13 \cdot 7$ & $24 \cdot 4$ \\
\hline SU64 & - & 54.7 & $13 \cdot 7$ & $\mathbf{2 5} \cdot \mathbf{0}$ \\
\hline SU91 & - & $52 \cdot 3$ & $13 \cdot 3$ & $25 \cdot 4$ \\
\hline SU 298/533 & $\mathbf{3 \cdot 8}$ & $47 \cdot 2$ & $11 \cdot 8$ & $\mathbf{2 5} \cdot 0$ \\
\hline NA30 & $2 \cdot 7$ & $50 \cdot 4$ & $15 \cdot 2$ & $30 \cdot 2$ \\
\hline NZ19 & $\mathbf{2 \cdot 9}$ & $48 \cdot 8$ & $16 \cdot 6$ & $34 \cdot 0$ \\
\hline $\begin{array}{l}\text { NZ19, from second } \\
\text { washing of cells }\end{array}$ & $1 \cdot 7$ & $53 \cdot 2$ & $17 \cdot 8$ & $33 \cdot 4$ \\
\hline $\begin{array}{l}\text { NZ 19, precipitated } \\
\text { thrice }\end{array}$ & $\mathbf{2 \cdot 0}$ & $58 \cdot 6$ & $19 \cdot 2$ & $32 \cdot 8$ \\
\hline $\begin{array}{l}\text { NZ19, insoluble matter } \\
\text { removed, precipitated } \\
\text { thrice }\end{array}$ & $1 \cdot 1$ & $61 \cdot 4$ & $20 \cdot 9$ & $34 \cdot 0$ \\
\hline $\begin{array}{l}\text { NZ19, Sevag-deprotein- } \\
\text { ized once, precipitated } \\
\text { thrice }\end{array}$ & $1 \cdot 0$ & $62 \cdot 2$ & $20 \cdot 2$ & $32 \cdot 5$ \\
\hline \multicolumn{5}{|l|}{ R. leguminosarum } \\
\hline SU302 & $2 \cdot 7$ & $48 \cdot 8$ & $7 \cdot 6$ & $15 \cdot 6$ \\
\hline SU 331 & - & $53 \cdot 2$ & $16 \cdot 2$ & $30 \cdot 5$ \\
\hline \multicolumn{5}{|l|}{ R. phaseoli } \\
\hline NA 1 & 一 & $57 \cdot 3$ & 14.5 & $25 \cdot 4$ \\
\hline \multicolumn{5}{|l|}{ R. meliloti } \\
\hline SU27 & 一 & $52 \cdot 5$ & n.d.§ & n.d.\$ \\
\hline SU47 & - & $42 \cdot 4$ & n.d. & n.d. \\
\hline SU 62 & - & 24.5 & n.d. & n.d. \\
\hline SU74 & - & 34.5 & n.d. & n.d. \\
\hline SU76 & - & $26 \cdot 5$ & n.d. & n.d. \\
\hline SU 101 & - & $32 \cdot 5$ & n.d. & n.d. \\
\hline SU277 & $3 \cdot 0$ & $32 \cdot 4$ & n.d. & n.d. \\
\hline
\end{tabular}

* All gums were precipitated twice from ethanol unless otherwise stated.

† Total carbohydrate = glucose + glucuronic acid: .

¥ It seems probable that 4-0-methyl glucuronic acid would be included in this fraction.

$\S$ n.d. = not detected. 


\section{Quantitative analysis of gums}

The ash content of the gums varied between 4 and $12 \%$. About $12 \%$ moisture which remained in the gum after drying in vacuo was removed by heating the gum at $100^{\circ}$ for $2 \mathrm{hr}$. Ash, moisture, protein (nitrogen) and polysaccharide analyses accounted for up to $90 \%$ of the weight of the gums.

The nitrogen content of the gums decreased on reprecipitation with ethanol and the content of polysaccharide increased (Table 2). However, the ratio glucose: glucuronic acid remained constant during purification, and also from one batch of culture to the next; this ratio therefore appears to be characteristic of the strain grown under these conditions.

In confirmation of the chromatographic observations, analysis showed that glucuronic acid, if present at all in the gums of the three lucerne strains, was below the limit of detection. The absorption curve of these gums in the presence of carbazole + sulphuric acid showed a very slight maximum at $530 \mathrm{~m} \mu$, which may have been due to a trace of glucuronic acid (less than $0 \cdot 1 \%$ of the gum).

\section{Serology}

We were not able to obtain convincing evidence of precipitation of a gum from either Rhizobium trifolii SU 298/533 or from $\boldsymbol{R}$. leguminosarum SU 331 with the homologous antisera developed against whole organisms of the same strains. Some doubtful positives were obtained at relatively high concentrations of antigen and antiserum.

\section{DISCUSSION}

Cooper \& Preston (1937) reported greater yields of polysaccharides from agar than from liquid medium (using a lucerne extract + minerals + carbon source mixture). This was not the case in the present study. Preliminary experiments, in which the organisms were grown on agar covered with cellophan.(Bray, Schluchterer \& Stacey, 1944), showed lower gum production than: that obtained on Norris's defined liquid medium. Ash values for gums produced on Norris's medium were significantly lower than those found by Bray et al. (1944). This may be due to the absence of calcium salts from Norris's medium, since calcium is thought to combine with glucuronic acid in these polysaccharides (Cooper, Daker \& Stacey, 1938). A fully defined medium, as used in the present study, eliminates the introduction of extraneous carbohydrate.

De Leizaola \& Dedonder (1955) grew their organisms in a medium of mineral salts and glucose, with and without yeast autolysate; the gums studied chromatographically were those grown in the absence of the yeast autolysate: It is possible that the complete absence of glucuronic acid which these workers found with the pea, soya bean, clover and lupin strains was due to the absence of co-enzymes necessary for the production of giucuronic acid. The other sugars detected by these workers (rhamnose, xylose, arabinose, galactose, mannose) might conceivably have resulted from this disorganization of the mechanism for gum production, or from contamination of the gum 
preparations with cell walls and contents. Sparse practical details make it impossible to exclude the latter explanation. We did not detect fructose in any of the gums we studied (compare Kleczkowski \& Kleczkowski, 1952).

In the present study only the lucerne organisms deviated from the 'classical' picture of Rhizobium gum (Hopkins et al. 1931) in their lack of glucuronic acid. Strominger, Maxwell, Axelrod \& Kalckar (1957) described a uridine diphosphoglucose dehydrogenase in liver which, in the presence of co-enzyme I, produced uridine diphosphoglucuronic acid from uridine diphosphoglucose. This they postulated as a step in the synthesis of glucuronides and of polysaccharides containing glucuronic acid. Strominger \& Mapson (1957) described a similar enzyme in pea seedlings. Smith, Mills \& Harper (1957) compared capsulated and non-capsulated strains of Pneumococcus in an attempt to correlate uridine diphosphoglucose metabolism with ability to form a capsule. They concluded that failure occurred at some laterstage than in the provision of building blocks. It is possible that the failure of Rhizobium meliloti strains to produce gums containing glucuronic aeid may lie in the absence of such an enzyme system. This has not yet been demonstrated in Rhizobium, however.

Previous quantitative analyses on Rhizobium gums have all been performed by methods of carbon dioxide liberation which require at least five hundred times the quantity of polysaccharide used in our spectrophotometric method. Other spectrophotometric methods for glucuronic acid estimation (e.g. Hanson, Mills \& Williams, 1944) are not valid in the presence of glucose. Bowness (1957) used the carbazole method for the analysis of hyaluronic acid, heparin and other polysaccharides. Like the carbazole estimation of glucuronic acid, the anthrone estimation of glucose has the advantage that previous hydrolysis of the polysaccharide, with the dangers of destruction or incomplete hydrolysis, is not necessary.

Hopkins, Peterson \& Fred (1930) found with a lucerne organism that the uronic acid was 4-7\% of the gum (calculated on an ash-free basis); in two clover strains it was $22-\mathbf{2 5} \%$ and in a pea strain, 19-22\%. The fact that their strain of Rhizobium meliloti (Wisconsin 100) yielded so much less uronic acid than the clover and pea strains provides a measure of agreement with our own failure to find glucuronic acid in any of the seven strains of the same species. Cooper et al. (1938) found $23-25 \%$ uronic acid in a clover strain of Rhizobium. The analyses reported in Table 2 of our paper put the polysaccharides into four groups : (i) about $33 \%$ glucuronic acid in the total carbohydrate (R. trifolii, NA30 and NZ19; R. leguminosarum, SU 331); (ii) about $25 \%$ glucuronic acid in total carbohydrate $(R$. trifolii, SU 36, 46, 64, 91 and 298/533; $R$. phaseoli, NA 1); (iii) about $15 \%$ glucuronic acid in total carbohydrate (R. leguminosarum, SU302); (iv) glucuronic acid not detected (R. meliloti, SU 27, $47 ; 62 ; 74 ; 76 ; ; 101$ and 277 ).

It will be seen from this grouping that though the glucose:glucuronic acid ratio appeared to be constant for the strain that was investigated in more detail (NZ 19; Table 2); it cannot be considered characteristic of species as they are at present defined: In other words, apart from the distinctiveness of: the Rhizobium meliloti strains, there was no obvious relationship between 
the glucose:glucuronic acid ratios and invasive specificity. Although gums which have the same glucose:glucuronic acid ratio cannot on this evidence be considered identical, those with different ratios can certainly be regarded as different. Yet it might still be possible for such gums to contain a groupcharacteristic structure not revealed on over-all analysis. It does not appear from our precipitation tests, nor from those of Kleczkowski \& Kleczkowski (1952), that specific agglutinability has any constant relationship to the gum fraction. Moreover, the production of an apparently similar polysaccharide by five strains of $R$. trifolii (SU 36, SU 46, SU 64, SU91 and SU 298/533) is not reflected in their somatic cross agglutinability. Strains SU 36 and SU 64 share an antigen, as do SU 46 and SU 91, but there is no cross-agglutination between the two pairs; nor does SU 298/533 cross react with the other strains. Similarly the seven strains of $R$. meliloti include some which share a common agglutinogen and others which do not. The loss of gum in variants of SU 298 (as well as SU 297) does not prevent agglutination with antisera developed against the gummy types (Vincent, 1954) and vice versa (Bloomfield, private communication).

Work reported in this paper has been supported by the Commonwealth Bank (Rural Credits Division), the Wheat Industry Research Funds, and by the Research Committee of the University of Sydney. Our thanks are due to Dr R. G. Wake for nitrogen analyses.

\section{REFERENCES}

Ashwell, G. (1957). Colorimetric analysis of sugars. In Methods in Enzymology, Vol. 3, ed. by S. P. Colowick and N. O. Kaplan. New York: Academic Press.

Bowness, J. M. (1957). Application of the carbazole reaction to the estimation of glucuronic acid and glucose in some acidic polysaccharides and in urine. Biochem. J. 67, 295.

Bray, H. G., Schluchterer, E. \& Stacey, M. (1944). Enzyme formation and polysaccharide synthesis by bacteria. Biochem. $J .38,154$.

Cooper, E., Daker, W. \& STACEY, M. (1938). Enzyme formation and polysaccharide synthesis by bacteria. Biochem. J. 32, 1752.

Cooper, E. \& Preston, J. F. (1937). Polysaccharide synthesis by nitrogen-fixing organisms. J. Soc. chem. Ind., Lond. 56, 1 T.

de Leizaola, M. \& Dedonder, R. (1955). Étude de quelques polyoisides produits par des souches de Rhizobium. C.R. Acad. Sci., Paris, 240, 1825.

Dische, Z. (1947). A new specific colour reaction of hexuronic acids. J. biol. Chem. $167,189$.

Hanson, S. W. F., Milis, G. T. \& Williams, R. T. (1944). A study of the determination of glucuronic acid by the naphthoresorcinol test with a.photoelectric absorptiometer. Biochem. J. 38, 274.

Hopkins, E. W., Peterson, W. H. \& Fred, E. B. (1930). Composition of the gum produced by root nodule bacteria. J. Amer. chem. Soc. 52, 3659.

Hopkins, E. W., Peterson, W. H. \& Fred, E. B. (1931). Glucuronic acid, a con stituent of the gum of root nodule bacteria. J. Amer. chem. Soc. 53, 306.

KLeczkowski, J. \& KLEczkowski, A. (1952). The effect of specific polysaccharid from the host bacteria and of ribonuclease on the multiplication of Rhizobium phages. J. gen. Microbiol. 7, 340.

Norris, D. (1958). Rhizobium needs magnesium, not calcium. Nature, Lond. 182, 734. 
Schluchterer, E. \& STACEY, M. (1945). The capsular polysaccharide of Rhizobium radicicolum. J. chem. Soc. p. 776.

Seifter, S., Dayton, S., Novic, B. \& Muntwyler, E. (1950). Estimation of glycogen with anthrone reagent. Arch. Biochem. 25, 191.

Sevag, M. G., Lackman, D. B. \& Smoluens, J. (1935). The isolation of the components of streptococcal nucleoproteins in serologically active form. J. biol. Chem. 124, 425.

Smith, E. B., Mills, G. T. \& Harper, E. M. (1957). A comparison of the uridine phosphoglucosyl metabolism of capsulated and non-capsulated pneumococci. J., gen. Microbiol. 16, 426.

Strominger, J. L. \& Mapson, L. W. (1957). Uridine diphosphoglucose dehydrogenase in pea seedlings. Biochem. J. 66, 567.

Strominger; J. L., Maxwell, E. S., Axelrod, J. \& Kalckar, H. M. (1957). Enzymatic formation of uridine diphosphoglucuronic acid. J. biol. Chem. 224, 79.

Vincent, J. M. (1954). The root-nodule bacteria of pasture legumes. Proc. Linn. Soc. N.S.W. 79, iv.

(Received 25 March 1959) 